\title{
JORDAN HOMOMORPHISMS
}

\author{
BY \\ I. N. HERSTEIN
}

The study of additive mappings from one ring $R$ into another ring $R^{\prime}$ which preserve squares was initiated by Ancochea $[1 ; 2]$ in connection with problems arising in projective geometry. Kaplansky [6] and Hua [4] took the subject up from where Ancochea had left off and pushed his results further. Jacobson and Rickart [5] then proceeded to carry out an extensive study of such functions.

In this paper we are primarily concerned with the study of certain additive mappings onto prime rings and in all cases we assume, ab initio, that the characteristic of the rings occurring as image rings is different from 2 . In this case the various definitions of Jordan homomorphism all coincide; we define a Jordan homomorphism here as a mapping $\phi: R \rightarrow R^{\prime}$ such that $\phi(a+b)=\phi(a)+\phi(b)$ and such that $\phi(a b+b a)=\phi(a) \phi(b)+\phi(b) \phi(a)$ for all elements $a$ and $b$ in $R$. In the situation where $\phi$ is onto $R^{\prime}$ and where $R^{\prime}$ is a prime ring of characteristic larger than 3 we obtain the theorem that $\phi$ is either a homomorphism or an anti-homomorphism. Of interest is the specialization to the case $R=R^{\prime}$ where $R^{\prime}$ is either a simple ring or a primitive ring; here we obtain that any Jordan automorphism is either an automorphism or an anti-automorphism. These results are closely related to some of those occurring in [5] and extend a few of these. In the last portion of the paper we characterize additive mappings onto prime rings of large enough characteristic (or 0 ) which preserve $n$th powers.

The material in this paper is entirely self-contained; in order to keep it so we re-prove a few lemmas that occur in [5] (our Lemmas 2 to 4 ). We also make use, on a number of occasions, of a theorem on linearization due to Gerstenhaber in his Ph.D. thesis; this result by itself can be found in [3]. The use of this linearization theorem leads to certain identities, obtained by linearization, in a quick fashion but in every particular case where we make use of it, because of the low order of multi-linearity of the functions involved, the reader can avoid using the general result and can carry out a series of linearizations to obtain the desired end-product.

We define again: a mapping $\phi$ from one ring into another ring $R^{\prime}$ is said to be a Jordan homomorphism from $R$ into $R^{\prime}$ if

$$
\begin{aligned}
\phi(a+b) & =\phi(a)+\phi(b), \\
\phi(a b+b a) & =\phi(a) \phi(b)+\phi(b) \phi(a),
\end{aligned}
$$

Presented to the Society, February 26, 1955; received by the editors February 4, 1955. 
for all $a$ and $b$ in $R$. In case $R^{\prime}$ is not of characteristic 2 (i.e. $2 x^{\prime}=0$ implies $\left.x^{\prime}=0\right),(2)$ is equivalent to

$$
\phi\left(a^{2}\right)=\phi(a)^{2} .
$$

It is of course clear that every homomorphism and every anti-homomorphism is a Jordan homomorphism.

We assume throughout the paper that $\phi$ is a Jordan homomorphism of $R$ into $R^{\prime}$ and that $R^{\prime}$ is not of characteristic 2 . We say that a ring $R^{\prime}$ is of characteristic greater than $n$ if $n ! x^{\prime}=0$ implies $x^{\prime}=0$.

The following variation of a linearization lemma appearing in [3] will be useful.

Lemma 1. Let $f\left(x_{1}, x_{2}, \cdots, x_{n}\right)$ be multilinear (multi-additive) from one ring $R$ into another ring $R^{\prime}$, and suppose that $f(a, a, \cdots, a)=0$ for all $a \in R$. Then

$$
\sum_{\pi} f\left(x_{\pi(1)}, x_{\pi(2)}, \cdots, x_{\pi(n)}\right)=0
$$

where $\pi$ runs through the symmetric group of degree $n$ acting on the letters $1,2, \cdots, n$,

(2) $f(b, a, a, \cdots, a)+f(a, b, a, \cdots, a)+\cdots+f(a, a, \cdots, a, b)=0$

for all $a, b$ in $R$ if the characteristic of $R^{\prime}$ is greater than $n-1$.

We now prove

Lemma 2. For all $a, b \in R, \phi(b a b)=\phi(b) \phi(a) \phi(b)$.

Proof. Since $\phi$ is a Jordan homomorphism,

However

$$
\begin{aligned}
\phi\{(a b+b a) b+b(a b+b a)\}= & \phi(a b+b a) \phi(b)+\phi(b) \phi(a b+b a) \\
= & {[\phi(a) \phi(b)+\phi(b) \phi(a)] \phi(b) } \\
& +\phi(b)[\phi(a) \phi(b)+\phi(b) \phi(a)] .
\end{aligned}
$$

$$
\begin{aligned}
\phi\{(a b+b a) b+b(a b+b a)\} & =\phi\left(a b^{2}+b^{2} a+2 b a b\right)=\phi\left(a b^{2}+b^{2} a\right)+2 \phi(b a b) \\
& =\phi(a) \phi(b)^{2}+\phi(b)^{2} \phi(a)+2 \phi(b a b) .
\end{aligned}
$$

Here we have made use of $\phi\left(b^{2}\right)=\phi(b)^{2}$. Comparing these two expressions we obtain $2 \phi(b a b)=2 \phi(b) \phi(a) \phi(b)$, and since $R^{\prime}$ is not of characteristic 2, $\phi(b a b)=\phi(b) \phi(a) \phi(b)$.

Linearizing Lemma 2 by replacing in it $b$ by $b+c$ we obtain

Lemma 3. For all $a, b, c \in R, \phi(a b c+c b a)=\phi(a) \phi(b) \phi(c)+\phi(c) \phi(b) \phi(a)$.

We can now establish

Lemma 4. For all $a, b \in R$, 


$$
\begin{aligned}
& {[\phi(a b)-\phi(a) \phi(b)][\phi(a b)-\phi(b) \phi(a)]=0} \\
& {[\phi(a b)-\phi(b) \phi(a)][\phi(a b)-\phi(a) \phi(b)]=0 .}
\end{aligned}
$$

Proof. We prove only (1) since the proof of (2) is exactly the same. Now

$$
\begin{aligned}
{[\phi(a b)-\phi(a) \phi(b)] } & {[\phi(a b)-\phi(b) \phi(a)] } \\
& =\phi(a b)^{2}+\phi(a) \phi(b)^{2} \phi(a)-\phi(a) \phi(b) \phi(a b)-\phi(a b) \phi(b) \phi(a) \\
& =\phi\left((a b)^{2}\right)+\phi\left(a b^{2} a\right)-\phi(a b(a b)+(a b) b a)
\end{aligned}
$$

(by Lemmas 2 and 3 )

$$
=\phi\left(a b a b+a b^{2} a-a b a b-a b^{2} a\right)=\phi(0)=0,
$$

proving the lemma.

We consider some consequences of Lemma 4.

Lemma 5. For all $a, b, r \in R$ $[\phi(a b)-\phi(a) \phi(b)] \phi(r)[\phi(a b)-\phi(a) \phi(b)]=[\phi(a b)-\phi(a) \phi(b)] \phi\{(a b-b a) r\}$.

Proof.

$$
\begin{aligned}
\phi(r)[\phi(a b) & -\phi(a) \phi(b)] \\
= & \phi(r) \phi(a b)-\phi(r) \phi(a) \phi(b) \\
= & \phi(r) \phi(a b)+\phi(b) \phi(a) \phi(r)-\phi(r a b+b a r) \quad \text { by Lemma } 3 \\
= & \phi(r) \phi(a b)+\phi(b) \phi(a) \phi(r)-\phi\{r(a b)+(a b) r\}+\phi\{(a b-b a) r\} \\
= & \phi(r) \phi(a b)+\phi(b) \phi(a) \phi(r)-\phi(r) \phi(a b)-\phi(a b) \phi(r)+\phi\{(a b-b a) r\} \\
= & \quad \text { (since } \phi \text { is a Jordan homomorphism) } \\
= & {[\phi(b) \phi(a)-\phi(a b)] \phi(r)+\phi\{(a b-b a) r\} . }
\end{aligned}
$$

Left multiplying both sides of this equation by $[\phi(a b)-\phi(a) \phi(b)]$ and making use of Lemma 4, we obtain

$$
[\phi(a b)-\phi(a) \phi(b)] \phi(r)[\phi(a b)-\phi(a) \phi(b)]=[\phi(a b)-\phi(a) \phi(b)] \phi\{(a b-b a) r\},
$$

which is the statement of Lemma 5 .

We multiply the result of Lemma 5 from the right by $[\phi(a b)-\phi(b) \phi(a)]$; using Lemma 4 we obtain

Lemma 6. For all $a, b, r \in R$

$$
[\phi(a b)-\phi(a) \phi(b)] \phi\{(a b-b a) r\}[\phi(a b)-\phi(b) \phi(a)]=0 .
$$

In Lemma 6 we replace $r$ by $r(a b-b a)$. Since by Lemma 2

$$
\phi(a b-b a) \phi(r) \phi(a b-b a)=\phi\{(a b-b a) r(a b-b a)\},
$$

Lemma 6 yields 
Lemma 7. For all $a, b, r \in R$

$$
[\phi(a b)-\phi(a) \phi(b)] \phi(a b-b a) \phi(r) \phi(a b-b a)[\phi(a b)-\phi(b) \phi(a)]=0 .
$$

A ring $R^{\prime}$ is said to be a prime ring if in $R^{\prime}, x^{\prime} R^{\prime} y^{\prime}=(0)$ implies that either $x^{\prime}=0$ or $y^{\prime}=0$. Every simple ring (radical or otherwise) and every primitive ring is a prime ring. Lemma 6 immediately implies

THEOREM A. Let $\phi$ be a Jordan homomorphism of a ring $R$ onto the prime ring $R^{\prime}$. Then for every $a, b$ in $R$ either

$$
[\phi(a b)-\phi(a) \phi(b)] \phi(a b-b a),=0
$$

or

$$
\phi(a b-b a)[\phi(a b)-\phi(b) \phi(a)]=0 .
$$

Let us consider the first possibility, namely that

$$
[\phi(a b)-\phi(a) \phi(b)] \phi(a b-b a)=0 .
$$

We evaluate $\phi(a b-b a)$;

$$
\begin{aligned}
\phi(a b-b a) & =2 \phi(a b)-\phi(a b+b a)=2 \phi(a b)-[\phi(a) \phi(b)+\phi(b) \phi(a)] \\
& =[\phi(a b)-\phi(a) \phi(b)]+[\phi(a b)-\phi(b) \phi(a)] .
\end{aligned}
$$

Left multiplying this by $\phi(a b)-\phi(a) \phi(b)$ and using Lemma 4 we then have

$$
0=[\phi(a b)-\phi(a) \phi(b)] \phi(a b-b a)=[\phi(a b)-\phi(a) \phi(b)]^{2} .
$$

Similarly if $\phi(a b-b a)[\phi(a b)-\phi(b) \phi(a)]=0$ we obtain that $[\phi(a b)-\phi(b) \phi(a)]^{2}$ $=0$.

We summarize these remarks as

THEOREM B. If $\phi$ is a Jordan homomorphism of a ring $R$ onto a prime ring $R^{\prime}$, then for any pair of elements $a$ and $b$ in $R$ at least one of $[\phi(a b)-\phi(a) \phi(b)]^{2}$ $=0$ or $[\phi(a b)-\phi(b) \phi(a)]^{2}=0$ must hold.

It will be convenient for us to change notation at this point. For $a, b \in R$ we let $a^{b}=\phi(a b)-\phi(a) \phi(b)$ and $a_{b}=\phi(a b)-\phi(b) \phi(a)$. We list a few elementary properties of the symbolism just introduced.

Lemma 8. For all $a, b, c \in R$

$$
\begin{aligned}
a^{b+c} & =a^{b}+a^{c}, \quad a_{b+c}=a_{b}+a_{c}, \\
(a+b)^{c} & =a^{c}+b^{c}, \quad(a+b)_{c}=a_{c}+b_{c}, \\
a^{b} & =-b^{a}, \quad a_{b}=-b_{a}, \\
a^{2 b} & =2 a^{b}, \quad a_{2 b}=2 a_{b}, \quad a^{-b}=-a^{b}, \quad a_{-b}=-a_{b} .
\end{aligned}
$$

The proofs of these are all immediate. Of course (4) is a special case of (1) but we point it out for emphasis; (3) is nothing but a restatement of the 
fact that $\phi$ is a Jordan homomorphism. We are now also able to apply Lemma 1 to $a^{b}$ and $a_{b}$ as linear functions from $R$ into $R^{\prime}$.

We assume henceforth throughout the paper that $R^{\prime}$ is a prime ring and that $\phi$ is a Jordan homomorphism of $R$ onto $R^{\prime}$.

In terms of this new notation, Lemma 4 becomes: for all $a, b \in R, a^{b} a_{b}$ $=a_{b} a^{b}=0$. Theorem B becomes: for all $a, b \in R$ at least one of $\left(a^{b}\right)^{2}=0$ or $\left(a_{b}\right)^{2}=0$. Thus

Lemma 9. For all $a, b, r \in R,\left(a_{b}\right)^{2} \phi(r)\left(a^{b}\right)^{2}=0$; that is $\left(a_{b}\right)^{2} R^{\prime}\left(a^{b}\right)^{2}=(0)$.

Consider the multi-additive function $f(x, y, u, z)$ from $R$ to $R^{\prime}$ defined by $f(x, y, u, z)=a_{x} a_{y} \phi(r) a^{u} a^{z}$; by Lemma $9 f(b, b, b, b)=0$ for all $b \in R$. So we can apply Lemma 1 . Since $f$ is multi-additive in four variables, to apply the second part of Lemma 1 we must assume that the characteristic of $R^{\prime}$ is greater than 3 . We consequently assume that indeed the characteristic of $R^{\prime}$ is larger than 3; the full power of Lemma 1 thus becomes available to us. Using Lemma 1 with $x=y=b$ and $u=z=c$ we obtain

$$
\left(a_{b}\right)^{2} \phi(r)\left(a^{c}\right)^{2}+\left(a_{c}\right)^{2} \phi(r)\left(a^{b}\right)^{2}+\left(a_{b} a_{c}+a_{c} a_{b}\right) \phi(r)\left(a^{b} a^{c}+a^{c} a^{b}\right)=0 .
$$

Using Lemma 1 with $x=y=u=b, z=c$, we have on simplifying and regrouping

$$
\left(a_{b}\right)^{2} \phi(r)\left(a^{b} a^{c}+a^{c} a^{b}\right)+\left(a_{b} a_{c}+a_{c} a_{b}\right) \phi(r)\left(a^{b}\right)^{2}=0 .
$$

Suppose that $\left(a_{b}\right)^{2} \neq 0$. By Theorem $\mathrm{B},\left(a^{b}\right)^{2}=0$. Thus (ii) reduces to

$$
\left(a_{b}\right)^{2} \phi(r)\left(a^{b} a^{c}+a^{c} a^{b}\right)=0 \quad \text { for all } r \in R \text {. }
$$

Since $R^{\prime}$ is a prime ring and since $\left(a_{b}\right)^{2} \neq 0$, it must follow that $a^{b} a^{c}+a^{c} a^{b}=0$ for all $c \in R$. This, together with $\left(a^{b}\right)^{2}=0$, reduces (i) to $\left(a_{b}\right)^{2} \phi(r)\left(a^{c}\right)^{2}=0$. The primeness of $R^{\prime}$ and the fact that $\left(a_{b}\right)^{2} \neq 0$ then forces $\left(a^{c}\right)^{2}=0$ for all $c \in R$. We have thus proved

Theorem C. If $R^{\prime}$ is of characteristic larger than 3 and if $\left(a_{b}\right)^{2} \neq 0$ for some $b \in R$, then $\left(a^{c}\right)^{2}=0$ for all $c \in R$.

Similarly, if $\left(a^{b}\right)^{2} \neq 0$ for some $b \in R$, it would have followed that $\left(a_{c}\right)^{2}=0$ for all $c \in R$.

The procedure we are to follow is to show that if $\left(a^{c}\right)^{2}=0$ for all $c \in R$ then $a^{c}=0$ for all $c$, and similarly if $\left(a_{c}\right)^{2}=0$ for all $c$ then $a_{c}=0$. So we shall assume that $\left(a_{b}\right)^{2} \neq 0$ for some pair of elements $a$ and $b$ in $R$. Our next theorem will then show that $\left(x^{y}\right)^{2}=0$ for all $x$ and $y$ in $R$ and eventually we shall prove that this forces $x^{y}=0$ holds universally in $R$. We could carry out the similar argument to prove that if $\left(a^{b}\right)^{2} \neq 0$ for some $b \in R$ then $\left(a_{c}\right)^{2}=0$ for all $c \in R$. In case $\left(a_{b}\right)^{2}=0$ for all $b$ we would eventually prove $a_{b}=0$. If $\left(a_{b}\right)^{2}=0$ and $\left(a^{b}\right)^{2}=0$ for all $a$ and $b$ in $R$, the reduction used in proving that $a_{b}=0$ (or $a^{b}=0$ ) would merely become easier. 
We assume henceforth that $R^{\prime}$, in addition to being a prime ring, is of characteristic larger than 3.

We proceed with

THEOREM D. If for some $a, b \in R,\left(a_{b}\right)^{2} \neq 0$ then $\left(x^{y}\right)^{2}=0$ for all $x$ and $y$ in $R$.

Proof. Suppose that $\left(a_{b}\right)^{2} \neq 0$. By Theorem C, of course, $\left(a^{x}\right)^{2}=0$ for all $x$ in $R$. Suppose that $\left(c^{d}\right)^{2} \neq 0$ for some $c, d \in R$. By Theorem $\mathrm{C}$ again, $\left(c_{x}\right)^{2}=0$ for all $x$ in $R$. Consider the element $a+c$. Now, either $\left[(a+c)^{x}\right]^{2}=0$ for all $x$, or $\left[(a+c)_{x}\right]^{2}=0$ for all $x$ in $R$. Suppose, say, that $\left[(a+c)^{x}\right]^{2}=0$ for all $x \in R$. Thus, since $\left(a^{x}\right)^{2}=0, a^{x} c^{x}+c^{x} a^{x}+\left(c^{x}\right)^{2}=0$. We claim that it is impossible that $\left[(a-c)^{x}\right]^{2}=0$ for all $x$ in $R$; for if $\left[(a-c)^{x}\right]^{2}=0$ for every $x$, this would result in $-a^{x} c^{x}-c^{x} a^{x}+\left(c^{x}\right)^{2}=0$, which when added to the result above would yield that $2\left(c^{x}\right)^{2}=0$, and so $\left(c^{x}\right)^{2}=0$ for all $x$ in $R$, contradicting that $\left(c^{d}\right)^{2} \neq 0$. Thus by Theorem $\mathrm{C}$, if $\left[(a+c)^{x}\right]^{2}=0$ then $\left[(a-c)_{x}\right]^{2}=0$ for all $x$ in $R$. If, on the other hand, $\left[(a+c)_{x}\right]^{2}=0$ for all $x$ in $R$, then $\left(a_{x}\right)^{2}+a_{x} c_{x}+c_{x} a_{x}=0$ since $\left(c_{x}\right)^{2}=0$. We claim that it is impossible that $\left[(a-c)_{x}\right]^{2}=0$ for all $x$ for then $\left(a_{x}\right)^{2}-a_{x} c_{x}-c_{x} a_{x}=0$ would result, which added to the result obtained above would lead to $\left(a_{x}\right)^{2}=0$ for all $x$ in $R$ contrary to $\left(a_{b}\right)^{2} \neq 0$. Also, since $(-c)^{d}$ $=-\left(c^{d}\right),\left((-c)^{d}\right)^{2} \neq 0$. Thus, without loss of generality we may assume that $\left[(a+c)^{x}\right]^{2}=0$ for all $x$ in $R$ and that $\left[(a-c)_{x}\right]^{2}=0$ for all $x$ in $R$. Thus $a^{x} c^{x}+c^{x} a^{x}+\left(c^{x}\right)^{2}=\left(a_{x}\right)^{2}-a_{x} c_{x}-c_{x} a_{x}=0$ for all $x \in R$. Now consider the element $a+2 c$. If $\left[(a+2 c)^{x}\right]^{2}=0$ for all $x \in R$ then $2\left(a^{x} c^{x}+c^{x} a^{x}\right)+4\left(c^{x}\right)^{2}=0$. Combined with $a^{x} c^{x}+c^{x} a^{x}+\left(c^{x}\right)^{2}=0$ this leads to $2\left(c^{x}\right)^{2}=0$ and so $\left(c^{x}\right)^{2}=0$ for all $x$ in $R$, contrary to assumption. If, on the other hand, $\left[(a+2 c)_{x}\right]^{2}=0$ then $\left(a_{x}\right)^{2}+2\left(a_{x} c_{x}+c_{x} a_{x}\right)=0$. Combined with $\left(a_{x}\right)^{2}-a_{x} c_{x}-c_{x} a_{x}=0$ this leads to $3\left(a_{x}\right)^{2}=0$, forcing $\left(a_{x}\right)^{2}=0$ for all $x$, contradicting $\left(a_{b}\right)^{2} \neq 0$. One of these two alternatives must always hold true, and yet each led to a contradiction. In this way the possibility that $\left(c^{d}\right)^{2} \neq 0$ is voided, and the theorem thereby proved.

We restate Theorem $\mathrm{D}$ as

\section{THEOREM D'. Either}

(a) $\left(x^{y}\right)^{2}=0$ for all $x$ and $y$ in $R$ or

(b) $\left(x_{y}\right)^{2}=0$ for all $x$ and $y$ in $R$.

The sequence of events to follow will show that if $\left(x^{y}\right)^{2}=0$ for all $x, y \in R$ then $\left(x^{v}\right)=0$. The obvious changes in the proof would show that if $\left(x_{y}\right)^{2}=0$ universally in $R$ then $\left(x_{y}\right)=0$. Since one or the other must hold, by Theorem $\mathrm{D}^{\prime}$, we proceed from here assuming that $\left(x^{y}\right)^{2}=0$ for all $x$ and $y$ in $R$.

Linearizing $\left(a^{b}\right)^{2}=0$ we obtain

Lemma 10. For all $a, b, c \in R, a^{b} a^{c}+a^{c} a^{b}=0$.

We change course for a short while. Our first turn is

Lemma 11. For all $a, x \in R, a^{\left(x^{2}\right)}=a^{x} \phi(x)+\phi(x) a^{x}$. 
Proof. $a^{\left(x^{2}\right)}=\phi\left(a x^{2}\right)-\phi(a) \phi\left(x^{2}\right)=\phi\left(a x^{2}\right)-\phi(a) \phi(x)^{2}$. But $\phi\left(a x^{2}+x a x\right)$ $=\phi(x) \phi(a x)+\phi(a x) \phi(x)$, therefore

$$
\phi\left(a x^{2}\right)=\phi(x) \phi(a x)+\phi(a x) \phi(x)-\phi(x) \phi(a) \phi(x) .
$$

Thus

$$
\begin{aligned}
\phi\left(a x^{2}\right)-\phi(a) \phi(x)^{2} & =\phi(x)[\phi(a x)-\phi(a) \phi(x)]+[\phi(a x)-\phi(a) \phi(x)] \phi(x) \\
& =a^{x} \phi(x)+\phi(x) a^{x} .
\end{aligned}
$$

Corollary. For all $x, y \in R, a^{x y+y x}=a^{x} \phi(y)+\phi(y) a^{x}+a^{y} \phi(x)+\phi(x) a^{y}$.

The proof of this corollary is immediate by linearizing Lemma 11.

We consider a special case of Lemma 10. In fact we have, by Lemma 10, that for $z=y^{2}, a^{y} a^{z}+a^{z} a^{y}=0$. Substituting for $a^{z}$ from Lemma 11 we have $\left[\phi(y) a^{y}+a^{y} \phi(y)\right] a^{y}+a^{y}\left[\phi(y) a^{y}+a^{y} \phi(y)\right]=0$. Since $\left(a^{y}\right)^{2}=0$ we obtain that $2 a^{y} \phi(y) a^{y}=0$, and so $a^{y} \phi(y) a^{y}=0$. That is

Lemma 12. For all $a, y \in R, a^{y} \phi(y) a^{y}=0$.

The linearized form of this lemma will be useful to us later so we single it out; we carry out the linearization using the second half of Lemma 1 . We obtain, in this way

Lemma 13. For all $a, x, y \in R$

$$
a^{y} \phi(x) a^{x}+a^{x} \phi(y) a^{x}+a^{x} \phi(x) a^{y}=0 .
$$

Our goal is to show that $b^{x}=0$ for all $b$ and $x$ in $R$. So we suppose that $b^{o} \neq 0$ for some $b, g$ in $R$.

Let $V^{\#}=\left\{u^{\prime} \in R^{\prime} \mid u^{\prime} b^{x}=b^{x} u^{\prime}=0\right.$ for all $\left.x \in R\right\}$ and let $V=\{u \in R \mid \phi(u)$ $\left.\in V^{\sharp}\right\}$. Let $W=\left\{x \in R \mid b^{x}=0\right\}$.

We now prove

LEMMA 14. $V \subset W$.

Proof. Suppose that $x \in V$. Thus for all $y \in R$, by Lemma $13, b^{x} \phi(x) b^{y}$ $+b^{x} \phi(y) b^{x}+b^{y} \phi(x) b^{x}=0$. However, since $x \in V, \phi(x) \in V^{\#}$, so $\phi(x) b^{y}=b^{y} \phi(x)$ $=0$. Thus the relation above reduces to $b^{x} \phi(y) b^{x}=0$ for all $y \in R$. Since $R^{\prime}$ is a prime ring, this forces $b^{x}=0$, and so $x \in W$. Thus $V \subset W$.

LEмma 15. $W$ is an additive subgroup of $R$, and if $2 x \in W$ then $x \in W$.

Proof. That $W$ is an additive subgroup of $R$ is clear. If $2 x \in W$, then $b^{2 x}=0$. However, by Lemma $8, b^{2 x}=2 b^{x}$; hence $2 b^{x}=0$. Since $R^{\prime}$ is of characteristic larger than 3 this implies that $b^{x}=0$. By the definition of $W$ this puts $x$ in $W$.

Lemma 16. If $u^{\prime} \in V^{\#}$ then for all $x, y \in R$

1. $u^{\prime} \phi(x) b^{x}=b^{x} \phi(x) u^{\prime}=0$, 
2. $u^{\prime} \phi(x) b^{y}+u^{\prime} \phi(y) b^{x}=0$,

3. $b^{y} \phi(x) u^{\prime}+b^{x} \phi(y) u^{\prime}=0$.

Proof. Let $z=x^{2}$. Thus $u^{\prime} b^{z}=b^{z} u^{\prime}=0$. However, by Lemma $11, b^{z}=\phi(x) b^{x}$ $+b^{x} \phi(x)$, so left multiplying this by $u^{\prime}$ we obtain $u^{\prime} \phi(x) b^{x}=0$. Similarly right multiplying by $u^{\prime}$ we have that $b^{x} \phi(x) u^{\prime}=0$. Linearizing these we establish parts (2) and (3) in the statement of the lemma.

Lemma 17. If $v \in V, r \in R$ then $v r+r v \in W$.

Proof. By the corollary to Lemma 11, $b^{r v+v r}=\phi(r) b^{v}+b^{v} \phi(r)+\phi(v) b^{r}$ $+b^{r} \phi(v)$. Since $v \in V$, by Lemma $14, v \in W$, and so $b^{v}=0$; since $v \in V, \phi(v) \in V^{*}$ hence $b^{r} \phi(v)=\phi(v) b^{r}=0$. Thus the right-hand side becomes 0 ; that is, $b^{r v+v r}=0$ putting $r v+v r$ in $W$.

LEмма 18. If $v \in V$, then for all $r \in R, b^{r v r}=0$; that is $r v r \in W$.

Proof.

$$
\begin{array}{rlr}
b^{2 r v r} & =b^{r(r v+v r)+(r v+v r) r}-b^{z v+v z} & \text { where } z=r^{2} \\
& =b^{r} \phi(r v+v r)+\phi(r v+v r) b^{r}+\phi(r) b^{r v+v r}+b^{r v+v r} \phi(r)-b^{z v+v z}
\end{array}
$$

by the corollary to Lemma 11 .

However the last three terms on the right-hand side are 0 by Lemma 17 . Thus

$$
\begin{aligned}
b^{2 r v r} & =b^{r} \phi(r v+v r)+\phi(r v+v r) b^{r} \\
& =b^{r} \phi(r) \phi(v)+b^{r} \phi(v) \phi(r)+\phi(r) \phi(v) b^{r}+\phi(v) \phi(r) b^{r}
\end{aligned}
$$

since $\phi$ is a Jordan homomorphism. Now $b^{r} \phi(v)=\phi(v) b^{r}=0$ since $v \in V$; on the other hand, $b^{r} \phi(r) \phi(v)=\phi(v) \phi(r) b^{r}=0$ by part (1) of Lemma 16. So $b^{2 r v r}=0$, whence $2 r v r \in W$ which implies that $r v r \in W$ by Lemma 15 .

Lemma 19. If $v \in V$ and $w \in W$ then $\phi(v) \phi(w) b^{x}=0$ for all $x \in R$.

Proof. By the part (2) of Lemma $16, \phi(v) \phi(w) b^{x}=-\phi(v) \phi(x) b^{w}=0$ since $b^{w}=0, w$ being in $W$.

Leмма 20. If $u^{\prime} \in V^{\sharp}$ then for all $r \in R, u^{\prime} \phi(r) u^{\prime} \phi(r) \in V^{\sharp}$.

Proof. $u^{\prime}=\phi(u)$, and where $u \in V$. But then Lemma 18 tells us that $r u r \in W$ for all $r$ in $R$, and so, by Lemma $19, \phi(u) \phi(r u r) b^{x}=0$ for all $x \in R$. That is, $\phi(u) \phi(r) \phi(u) \phi(r) b^{x}=0$; by the very definition, since $b^{x} \phi(u) \phi(r) \phi(u) \phi(r)$ is also $0, \phi(u) \phi(r) \phi(u) \phi(r) \in V^{\star}$, proving the lemma.

Suppose now that $u^{\prime} \in V^{\sharp} ;$ thus $u^{\prime} \phi(r) u^{\prime} \phi(r) b^{x}=0$ for all $r, x \in R$. Replacing $r$ in this by $r+s$ we obtain

Lемма 21. If $u^{\prime} \in V^{\sharp}$, then for all $r, s \in R$ and all $x \in R, u^{\prime} \phi(r) u^{\prime} \phi(s) b^{x}$ $+u^{\prime} \phi(s) u^{\prime} \phi(r) b^{x}=0$. 
We are now able to prove the key

Lemma 22. If $u^{\prime} \in V^{\sharp}$ and $b^{s} \neq 0$ (that is, $s \notin W$ ) then $u^{\prime} \phi(s) u^{\prime}=0$.

Proof. By Lemma 21, putting $x=s$ we have $u^{\prime} \phi(r) u^{\prime} \phi(s) b^{s}+u^{\prime} \phi(s) u^{\prime} \phi(r) b^{s}$ $=0$. However, by part (1) of Lemma $16, u^{\prime} \phi(s) u^{\prime} \phi(r) b^{s}=0$. Thus we obtain that $u^{\prime} \phi(s) u^{\prime} \phi(r) b^{s}=0$ for all $r \in R$. Since $b^{s} \neq 0$ and since $R^{\prime}$ is a prime ring, it follows that $u^{\prime} \phi(s) u^{\prime}=0$, which is the contention of the lemma.

We proceed from here to prove

Theorem E. If $u^{\prime} b^{x}=b^{x} u^{\prime}=0$ for all $x \in R$ then $u^{\prime}=0$; that is, $V^{\sharp}=(0)$.

Proof. By Lemma 22, if $s \notin W$ then $u^{\prime} \phi(s) u^{\prime}=0$. Suppose now that $w \in W, s \notin W$. Since $W$ is an additive subgroup of $R, s+w \in W$. So Lemma 22 yields again that $u^{\prime} \phi(s+w) u^{\prime}=0$. That is $u^{\prime} \phi(s) u^{\prime}+u^{\prime} \phi(w) u^{\prime}=0$. However, since $s \notin W, u^{\prime} \phi(s) u^{\prime}=0$. So we are left with $u^{\prime} \phi(w) u^{\prime}=0$ for all $w \in W$. Combined with Lemma 22 this yields that $u^{\prime} \phi(r) u^{\prime}=0$ for all $r \in R$. Since $R^{\prime}$ is a prime ring, this results in $u^{\prime}=0$, establishing Theorem $\mathrm{E}$.

Having shown that if $b^{0} \neq 0$ for some elements $b$ and $g$ in $R$ then we can find no element $u^{\prime}$ in $R^{\prime}$ such that $u^{\prime} b^{x}=b^{x} u^{\prime}=0$ for all $x$ in $R$, other than $u^{\prime}=0$, we proceed to construct such $u^{\prime}$ and to derive consequences from the fact that these elements must turn out to be 0 . This will be seen in the proof of

Theorem F. For all $b, y \in R, b^{y} \phi(y)=\phi(y) b^{y}$.

Proof. If $b^{z}=0$ for all $z \in R$, the theorem is, of course, trivially true. So let us suppose that $b^{0} \neq 0$ for some $g \in R$. By Lemma 10, for all $y, z \in R$,

$$
b^{y} b^{z}+b^{z} b^{y}=0 .
$$

In this relation we replace $z$ by $b z$. So we must evaluate $b^{b z}$. Now

$$
\begin{aligned}
b^{b z}= & \phi(b \cdot b z)-\phi(b) \phi(b z)=\phi\left(b^{2} z\right)-\phi(b) \phi(b z) \\
= & \phi(b) \phi(b z)+\phi(b z) \phi(b)-\phi(b z b)-\phi(b) \phi(b z) \\
& \quad \text { since } \phi \text { is a Jordan homomorphism } \\
= & \phi(b z) \phi(b)-\phi(b z b)=\phi(b z) \phi(b)-\phi(b) \phi(z) \phi(b) \\
= & {[\phi(b z)-\phi(b) \phi(z)] \phi(b)=b^{z} \phi(b) . }
\end{aligned}
$$

Thus, by replacing $z$ by $b z$ in $\left({ }^{*}\right)$ it becomes $b^{y} b^{z} \phi(b)+b^{z} \phi(b) b^{y}=0$. Using $\left(^{*}\right)$ in this we arrive at $-b^{z} b^{y} \phi(b)+b^{z} \phi(b) b^{y}=0$. That is, $b^{z}\left(b^{y} \phi(b)-\phi(b) b^{y}\right)=0$ for all $z \in R$. Similarly, using the substitution of $z b$ for $z$ in $\left({ }^{*}\right)$ we obtain $\left(b^{y} \phi(b)\right.$ $\left.-\phi(b) b^{y}\right) b^{z}=0$. Thus, by Theorem $\mathrm{E}, b^{y} \phi(b)-\phi(b) b^{y}=0$ for all $b$ and $y$ in $R$. However, $b^{y}=-y^{b}$ by Lemma 8 . So we have that $-y^{b} \phi(b)=-\phi(b) y^{b}$. Interchanging the labelling of $b$ and $y$ we have that $b^{y} \phi(y)=\phi(y) b^{y}$ for all $b$ and $y$ in $R$; this completes the proof of Theorem $\mathrm{F}$.

Theorem G. For all $b, z \in R, b^{2}=0$. 
Proof. If $t=z^{2}$, then, by Lemma $11, b^{t}=\phi(z) b^{z}+b^{z} \phi(z)$. Using the result of Theorem $\mathrm{F}$ this further simplifies to $b^{t}=2 \phi(z) b^{z}=2 b^{z} \phi(z)$. Thus $b^{t} b^{y}$ $=2 b^{z} \phi(z) b^{y}$ and $b^{v} b^{t}=2 b^{y} \phi(z) b^{z}$. However, by Lemma $10, b^{t} b^{y}+b^{y} b^{t}=0$; using the values of $b^{y} b^{t}$ and $b^{t} b^{y}$ just calculated, we have $2\left[b^{y} \phi(z) b^{z}+b^{z} \phi(z) b^{y}\right]=0$. In $R^{\prime}$ this implies that $b^{y} \phi(z) b^{z}+b^{z} \phi(z) b^{y}=0$ for all $b, y, z \in R$. However, by Lemma $13, b^{y} \phi(z) b^{z}+b^{z} \phi(y) b^{z}+b^{y} \phi(z) b^{z}=0$. In light of the above result, this reduces to $b^{z} \phi(y) b^{z}=0$ for all $y$ and $z$ in $R$. Since $R^{\prime}$ is a prime ring, $b^{z}=0$ for all $b, z \in R$, proving Theorem $\mathrm{G}$.

Thus we have proved that if $\left(x^{y}\right)^{2}=0$ holds for all elements $x$ and $y$ in $R$, then $x^{y}=0$ follows. Similarly, if $\left(x_{y}\right)^{2}=0$ holds universally in $R$, then $x_{y}=0$ follows. Since one of these two possibilities must prevail, we have indeed shown that either $x^{y}=0$ for all $x$ and $y$ in $R$ or $x_{y}=0$ for all $x$ and $y$ in $R$. The first alternative, from the definition of $x^{y}$, implies that $\phi$ is a homomorphism, the second alternative implies that $\phi$ is an anti-homomorphism. Thus we have proved the main result.

THEOREM H. If $\phi$ is a Jordan homomorphism of a ring $R$ onto a prime ring $R^{\prime}$ of characteristic different from 2 and 3 then either $\phi$ is a homomorphism or an anti-homomorphism.

Interesting corollaries of Theorem $\mathrm{H}$ come on its application to two special cases of prime rings, namely, simple rings and primitive rings. We record these as

THEOREM I. A Jordan automorphism of a simple ring of characteristic different from 3 and 2 is either an automorphism or an anti-automorphism.

THEOREM J. A Jordan automorphism of a primitive ring of characteristic different from 2 and 3 is either an automorphism or an anti-automorphism.

Theorem J of course implies Theorem I except in the possible (?) case of a simple radical ring, for which Theorem I still holds true.

In the concluding part of this paper we consider mappings, $\phi$, from one ring $R$ into another ring $R^{\prime}$ such that

$$
\begin{aligned}
\phi(a+b) & =\phi(a)+\phi(b), \\
\phi\left(a^{n}\right) & =\phi(a)^{n}
\end{aligned}
$$

for some fixed integer $n>2$. We shall call such mappings $n$-Jordan mappings. We prove the

THEOREM K. Let $\phi$ be an $n$-Jordan mapping from a ring $R$ onto a prime ring $R^{\prime}$ of characteristic larger than $n$. Suppose further that $R$ has a unit element. Then $\phi=\epsilon \tau$ where $\tau$ is either a homomorphism or an anti-homomorphism and where $\epsilon$ is an $(n-1)$ st root of unity lying in the center of $R^{\prime}$.

Proof. Since $\phi(x)^{n}=\phi\left(x^{n}\right)$, linearizing this and using Lemma 1 we have, 
where $\pi$ runs through the symmetric group of degree $n$,

$$
\sum_{\pi} \phi\left(x_{\pi(1)}, x_{\pi(2)}, \cdots, x_{\pi(n)}\right) \equiv \sum_{\pi} \phi\left(x_{\pi(1)}\right) \phi\left(x_{\pi(2)}\right) \cdots \phi\left(x_{\pi(n)}\right) .
$$

Let $\phi(1)=\alpha$ where 1 is the unit element of $R$. Thus $\phi(1)=\phi\left(1^{n}\right)=\phi(1)^{n}$, thus $\alpha^{n}=\alpha$. Putting $x_{1}=x, x_{2}=x_{3}=\cdots=x_{n}=1$ in (1) we obtain

$$
n ! \phi(x)=(n-1) !\left(\alpha^{n-1} \phi(x)+\alpha^{n-2} \phi(x) \alpha+\cdots+\phi(x) \alpha^{n-1}\right),
$$

and since the characteristic of $R^{\prime}$ is larger than $n$, this simplifies to

$$
n \phi(x)=\alpha^{n-1} \phi(x)+\alpha^{n-2} \phi(x) \alpha+\cdots+\alpha \phi(x) \alpha^{n-2}+\phi(x) \alpha^{n-1} .
$$

Using $\alpha^{n}=\alpha$ and multiplying (3) through from the right by $\alpha$ we have $n \phi(x) \alpha=\alpha^{n-1} \phi(x) \alpha+\alpha^{n-2} \phi(x) \alpha^{2}+\cdots+\phi(x) \alpha$. Multiplying through (3) by $\alpha$ from the left we obtain $n \alpha \phi(x)=\alpha \phi(x)+\alpha^{n-1} \phi(x) \alpha+\cdots+\alpha \phi(x) \alpha^{n-1}$. These lead by transposing and comparing to $(n-1) \alpha \phi(x)=(n-1) \phi(x) \alpha$. Since the characteristic of $R^{\prime}$ exceeds $n$, this forces $\phi(x) \alpha=\alpha \phi(x)$. Thus $\alpha$ is in the center of $R^{\prime}$. Thus (3) simplifies to $n \phi(x)=n \alpha^{n-1} \phi(x)$, and again from the fact that the characteristic of $R^{\prime}$ is larger than $n$ we are led to $\alpha^{n-1} \phi(x)=\phi(x)$. Thus $\alpha^{n-1}$ is the unit element of $R^{\prime}$ and $\alpha$ is an $(n-1)$ st root of unity lying in the center of $R^{\prime}$. We specialize in (1) by putting $x_{1}=x$, $x_{2}=y, x_{3}=\cdots=x_{n}=1$. We obtain, using that the characteristic of $R^{\prime}$ exceeds $n$, that $\phi(x y+y x)=\alpha^{n-2}[\phi(x) \phi(y)+\phi(y) \phi(x)]$. Let $\tau=\alpha^{n-2} \phi$; thus $\tau(x y+y x)=\alpha^{n-2} \phi(x y+y x)=\alpha^{n-2} \alpha^{n-2}[\phi(x) \phi(y)+\phi(y) \phi(x)]=\left(\alpha^{n-2} \phi(x)\right)\left(\alpha^{n-2} \phi(y)\right)$ $+\left(\alpha^{n-2} \phi(y)\right)\left(\alpha^{n-2} \phi(x)\right)=\tau(x) \tau(y)+\tau(y) \tau(x)$. Thus $\tau$ is a Jordan homomorphism, and so by Theorem H, $\tau$ is either a homomorphism or an anti-homomorphism. Since $\phi=\left(\alpha^{n-2}\right)^{-1} \tau$, and $\alpha^{n-2}$ is an $(n-1)$ st root of unity and lies in the center of $R^{\prime}$, Theorem $\mathrm{K}$ is completely proved.

One might conjecture that an appropriate variant of Theorem $\mathrm{K}$ would hold even if $R$ does not have a unit element.

\section{REFERENCES}

1. G. Ancochea, Le théorème de von Staudt en géometrie projective quaternionienne, J. Reine Angew. Math. vol. 184 (1942) pp. 192-198. 147-154.

2. - On semi-automorphisms of division algebras, Ann. of Math. vol. 48 (1947) pp.

3. M. Gerstenhaber, $A$ note on linearization (forthcoming).

4. L. K. Hua, On the automorphisms of a field, Proc. Nat. Acad. Sci. U.S.A. vol. 35 (1949) pp. 386-389.

5. N. Jacobson and C. E. Rickart, Jordan homomorphisms of rings, Trans. Amer. Math. Soc. vol. 69 (1950) pp. 479-502.

6. I. Kaplansky, Semi-automorphisms of rings, Duke Math. J. vol. 14 (1947) pp. 521-527.

University of Pennsylvania,

Philadelphia, Pa. 